\title{
CT Scanning in Minor Head Injury
}

\author{
Saeed Shoar and Soheil Saadat \\ Sina Trauma Research Center \\ Tehran University of Medical Sciences
}

Iran

\section{Introduction}

Head injury is one of the most prevalent events accounting for about one million of emergency visits in US and UK annually. It occurs in over the $50 \%$ of traumatic patients and is the leading cause of death and disabilities in children and younger adults all over the world (Holmes, et al., 2006; Kraus \& Nourjah, 1998; Langlois, et al., 2006).

Annually, traumatic brain injuries cause 435000 emergency department visits, 37000 hospital admissions, and 2685 deaths among children whose ages range from 0 to 14 in the USA (Jager et al., 2000; Langlois et al.,2004).

While there has been an increase in the number of CT performed for traumatic patients, particularly those with a suspected head injury, different studies have estimated the prevalence of significant intracranial lesions on a CT to be something between $0.7 \%$ and $20 \%$ (Bordignon \& Arruda 2002, Holmes et al., 2006, Mower 2005, Stiell 2001). Because most of these patients have insignificant injuries requiring no specific therapy, some authorities are reluctant to advocate CT studies in all such patients. In contrast, other authorities, concerned with the potentially dramatic consequences of a missed finding, tend to encourage the liberal use of brain CT in such patients bringing an estimated 750 million $\$$ cost for health system (Borczuk, 1995; Jeret et al., 1993; Schynoll et al., 1993); however, it has been reported that reduction in the number of patients complicated due to head trauma is happening as a result of better control of developing seizure or raised intracranial pressure (Klauber et al. 1989).

The majority of treated brain injuries are categorized as mild head injury (MHI) (Kraus \& Nourjah,1988). While patients with moderate to severe head injury usually show obvious clinical signs, simplifying the decision to perform a head CT scan, there are controversies in the indications of CT scanning in MHI patients considering the fact that most of these patients represent minimal intracranial lesions, requiring no specific therapy (Shackford et al., 1992). This chapter will recap the application of CT scanning in MHI patients in adults and children.

\section{Minor head injury}

The term "mild/minor head injury" was first described by Rimel and colleagues in 1981 (Rimel et al., 1981) as a head damage with rapid healing and not much severe posttraumatic complications. As the time past; however, more cases with fatal complications and further Sequelae due to MHI were detected. Between $6 \%$ to $21 \%$ of such patients develop intracranial lesions and $0.4 \%$ to $1 \%$ of them need neurosurgical intervention (Miller et al., 1997; af Geijerstam \& Britton, 2003; Borg et al., 2004; Fabbri et al., 2004; Haydel, et al., 2000). 
Having a minor nature of presentation in emergency admission, minor head injury is defined as a history of loss of consciousness $<15-20$ minutes, amnesia $<1$ hour, or disorientation in a conscious and talking patient, that is, one with Glasgow Coma Scale (GCS) of 13-15. These patients may experience fatigue, dizziness, difficulty in concentration and performing mental tasks, irritability, impairment of memory, insomnia and, decreased tolerance to stress, altogether referred to as "post- concussional syndrome" (PCS). Patients with "minimal head injury" may not experience an LOC or other neurological alteration (Stiell et al., 2005). Traumatic patients' visits start with determination of GCS in initial assessment. This scale is apparently correlated with severity of the damage happening inside the skull and beside; it can be measured with sufficient reliability by the health care providers (Menegazzi et al., 1993, Norwood et al., 2002). Therefore, GCS is a widely accepted measure of severity of neurological trauma.

There are variations in the definition of such injury; from a history of blunt head trauma on the initial emergency department evaluation with no experience of loss of consciousness or other neurologic deficits, to some more serious events causing higher impact on patient's alertness.

The incidence of hospital-treated patients with mild traumatic brain injury is something between 100 and 300 cases per 100,000 populations. Note, however, that much mild traumatic brain injury is not treated at hospitals, and the true population-based rate is probably more than 600 cases per 100,000 populations. The estimated number of patients with head trauma in the US ranges from 800,000 to 2 million cases annually. Of these cases, more than $80 \%$ are classified as minor head injuries (Cassidy et al., 2004).

\section{CT scan in head trauma}

Computed tomography (CT) is the modality of choice in trauma centers which helps rapid and accurate diagnosis of damages occurred to the head from a simple skull fracture to more serious lesions such as intracranial hematoma, hemorrhage, and brain contusions. From the emergence of CT scanning in medicine in 1970s, it has brought many benefits to patients. Quick diagnosis of ongoing intra cranial damage and the possible neurosurgical intervention afterward is the key to overcome life threatening events in head injured patients (Shackford et al., 1992; Stein \& Ross, 1990, 1992). However, its overuse and the side effects of radiation on patients has been a concern. In 1990s, studies reported that positive findings in CTs performed during management of patients, did not exceed of $20 \%$ of traumatic cases (Stein \& Ross, 1990; Harad \& Kerstein 1992). However, the number of ordered CT scan increased by $120 \%$ and reached to $5.3 \%$ from the previous $2.4 \%$ in all emergency department visits. Currently in the US, 1 million patients with blunt head trauma undergo head CT scanning annually (Mc Caig \& Burt 2001). This shows that CT has been increasingly overused in the past decade, while its diagnostic benefits have remained low.

CT scanning is generally repeated in order to follow traumatic patients and this increase the radiation concerns. Reporting the collective effective dose of radiation in CT scanning as $60 \%$ (2005-2006) in UK, 82\% in USA, and 67\% in Germany (2000-2005), it was showed that CT has been responsible for more than half of the radiation due to diagnostic imaging (Berrington De Gonzalex \& Darby, 2004; Brix, 2009; Einstein, 2007; Hall \& Brenner, 2008; Mettler, 2000).

Coming to the economic world, despite its wide spreading and explosive utilization, CT is an expensive modality in many countries (Katada, 2006). Besides its economic burden, additional benefits from mindful use of CT would include saving time, reducing overcrowding in the emergency, neurology, and radiology departments, decreased 
radiation, no more need for applying sedation in children, and preventing unnecessary transfers from departments without access to CT, like rural trauma centers, which in turn may harm critically ill patients.

\section{The application of decision rules to order CT scanning}

Prediction rules that help physicians to identify patients with clinically significant lesions on CT would reduce the number of scans performed and save millions of dollars in unnecessary scans. A $10 \%$ reduction in the number of scans ordered for minor head injury could result in a \$20 million reduction in healthcare expenditures in the US (Haydel, 2005). In fact, it is rational to screen patients of mild head injury who have high risk factors to develop intracranial lesions which in turn affects the final result. Such risk factors include: amnesia, loss of consciousness, vomiting, possible skull fracture, history of coagulopathy or using anticoagulant, post-traumatic seizures, asymmetry in pupils, severe or increasing headache, focal neurological deficits, or multiple trauma (Dunning et al., 2004).

There are several decision rules that are developed to identify those MHI patients who will probably benefit from head CT scanning; from the earliest known "New Orleans Criteria (NOC)" in 2000, to recently defined CATCH rule (2010), for "Canadian Assessment of Tomography for Childhood Head injury". The other published decision rules include: "Canadian CT Head Rule (CCHR)", "CT in Head Injury Patients (CHIP) rule" , "Prediction of intracranial computed tomography findings in patients with minor head injury by using logistic regression" and "CATCH rule for Canadian Assessment of Tomography for Childhood Head injury" (Saadat et al., 2009; Smits et al., 2007; Stiell et al., 2001).

Being among the first prospectively designed decision rules, NOC and CCHR have been used extensively. These criteria are claimed to identify traumatic patients who need neurosurgical intervention after a minor head injury with $100 \%$ sensitivity; thus there is no need to perform CT scanning for MHI patients who do not represent sign and symptoms described in the decision rule. "New Orleans Criteria (NOC)" and "Canadian CT head rule (CCHR)" have been validated by Smith et al., in 2005 (smith et al., 2005). CT in head injury patients (CHIP) rule was developed by smith et al., after validation of NOC and CCHR.

It is clear that the highest possible sensitivity is required to avoid an unwanted neglect during emergency observation of MHI patients. In a survey carried out by Graham (Graham et al., 1998), more than half of the respondents among emergency physicians insisted on the necessity of a $100 \%$ sensitivity for the clinical decision guidelines which is aimed to be disseminated for ordering CT scan in mild head injury patients. However, the sensitivity and specificity that are reported in the original reports, fail to remain at the same level in other studies. Moreover, the external validity of the above mentioned decision rules are not exactly the same as they have included a specific group of MHI patients.

\section{Indications of CT scan in minor head injury (MHI) patients}

\subsection{Miller's criteria, 1997}

It was around 1997, when Miller et al. introduced almost the first clinical criteria to determine whether it could safely eliminate the need to send all the patients sustained a head trauma for head CT scanning (Miller et al., 1997).

They included all patients with a normal mental status, a history of LOC or amnesia, and who presented less than 2 hours after blunt head trauma to the emergency department. The 
patients included in Miller study were awake (i.e. having a GCS score of 15) at the time of presentation to the emergency department.

The positive outcome in CT scan included contusion, parenchymal hematoma, epidural hematoma, subdural hematoma, subarachnoid hemorrhage or a skull fracture.

The study sample size was 2143 . They proposed that patients having one or more of the following signs or symptoms would need head CT scanning: severe headache, nausea, vomiting, and depressed skull fracture. While their criteria identified most of the minor head trauma patients with abnormal CT scan and all of those who need neurosurgical intervention, it reduced the number of performed head CT scans up to $61 \%$.

\subsection{New Orleans Criteria (NOC), 2000}

The New Orleans Criteria (NOC) was introduced by Haydel et al. in 2000. They first developed their criteria by reviewing $520 \mathrm{MHI}$ patients and then validated it by applying the criteria on other 909 patients.

They included all patients with a history of head injury, older than 3 years who presented within 24 hours to emergency department, and had a loss of consciousness with normal findings on a brief neurologic examination (normal cranial nerves and normal strength and sensation in the arms and legs) and a score of 15 on the Glasgow Coma Scale. Patients with previous CT, and no history of loss of consciousness (LOC) or amnesia were excluded.

The positive outcome included subdural, epidural, or parenchymal hematoma; subarachnoid hemorrhage; cerebral contusion; or depressed skull fracture.

The study sample size was 1429 .

\begin{tabular}{|c|c|c|c|c|c|c|c|c|}
\hline & Miller & NOC & CCHR & \begin{tabular}{|l} 
Abdul \\
Latip
\end{tabular} & NEXUS II & CHIP & Lee & Saadat \\
\hline $\begin{array}{l}\text { Age group } \\
\text { included }\end{array}$ & $\begin{array}{l}\text { Adults and } \\
\text { children }\end{array}$ & $>3$ & $\geq 16$ & $\geq 12$ & ? & $\geq 16$ & $\geq 16$ & $15-70$ \\
\hline GCS included & 15 & 15 & $13-15$ & $13-15$ & 15 & \begin{tabular}{|l}
$13-14$ \\
15 if \\
accompanied \\
by another risk \\
factor
\end{tabular} & $13-15$ & $13-15$ \\
\hline $\begin{array}{l}\text { Was LOC } \\
\text { necessary to be } \\
\text { included in the } \\
\text { study? }\end{array}$ & Yes & Yes & Yes & No & ? & $\begin{array}{l}\text { If there was no } \\
\text { loss of GCS }\end{array}$ & No & No \\
\hline $\begin{array}{l}\text { CT scan } \\
\text { performed for all } \\
\text { patients? }\end{array}$ & Yes & Yes & No & ? & No & $\begin{array}{l}\text { According to } \\
\text { the guideline } \S\end{array}$ & ? & Yes \\
\hline Sample size & 2143 & $520+909$ & 3121 & 94 & 2100 & 3181 & 898 & 318 \\
\hline $\begin{array}{l}\text { Included } \\
\text { patients if MHI } \\
\text { had occurred } \\
\text { during the past: }\end{array}$ & $2 \mathrm{~h}$ & $24 \mathrm{~h}$ & $24 \mathrm{~h}$ & $?$ & $?$ & $24 \mathrm{~h}$ & $12 \mathrm{~h}$ & $12 \mathrm{~h}$ \\
\hline
\end{tabular}

? Not specifically described in the article

§ Twijnstra A, Brouwer O, Keyser A, Lanser J, Poels E, Rinkel G, et al. Guidelines for diagnosis and management of patients with minor head injury. Published in Dutch: Richtlijnen voor diagnostiek en behandeling van patie"nten met een licht schedel-hersenletsel. Accessed at www.neurologie.nl/richtlijnen Accessed on 15 December 2006.

Table 1. Comparison of external validity of different criteria for brain CT scan in adulthood 
The New Orleans Criteria consists of the following finding (Haydel et al., 2000):

1. Headache

2. Vomiting

3. Age more than 60

4. Drug or alcohol intoxication

5. Persistent antegrade amnesia (short- term memory deficit)

6. Visible trauma above the clavicle

7. Seizure

When using all the findings together, a $22 \%$ reduction would be anticipated to be achieved. The sensitivity and specificity of NOC was reported as $100 \%$ and $12.7 \%$, respectively.

\subsection{Canadian CT Head Rule (CCHR), 2001}

Stiell et al. developed the Canadian CT Head Rule (CCHR) by studying $3121 \mathrm{MHI}$ patients (stiell et al., 2001, 2005).

They included patients with a history of blunt trauma to the head resulting in witnessed loss of consciousness, definite amnesia, or witnessed disorientation; initial emergency department GCS score of 13 or greater; who were injured during the past 24 hours. They excluded the following patients: patients younger than 16 years old; minimal head injury (i.e., no loss of consciousness, amnesia, or disorientation); no clear history of trauma as the primary event (e.g., primary seizure or syncope); an obvious penetrating skull injury or obvious depressed fracture, had acute focal neurological deficit; had unstable vital signs associated with major trauma; had a seizure before assessment in the emergency department; had a bleeding disorder or used oral anticoagulants (i.e., coumadin); had returned for reassessment of the same head injury; or were pregnant.

The positive findings in the CT scan were defined in two categories: findings that necessitated neurological intervention and findings that indicated clinically important brain injury on CT. Need for neurological intervention was defined as either death within 7 days secondary to head injury or the need for any of the following procedures within 7 days: craniotomy, elevation of skull fracture, intracranial pressure monitoring, or intubation for head injury (shown on CT). Clinically important brain injury was defined as any acute brain finding revealed on CT which would normally require admission to hospital and neurological follow-up.

The following lesions were not considered a positive finding if the patient was neurologically intact: solitary contusion less than $5 \mathrm{~mm}$ in diameter; localized subarachnoid blood less than $1 \mathrm{~mm}$ thick; smear subdural hematoma less than $4 \mathrm{~mm}$ thick; isolated pneumocephaly, or closed depressed skull fracture not through the inner table.

Not all patients necessarily underwent CT scanning but based on the judgment of the treating physician.

CCHR is consisted of five high- risks and 2 medium- risks criteria. The high risk criteria are capable of detecting intracranial lesions that are severe enough to necessitate a neurological intervention, while medium risk criteria identify patients who probably have sustained brain injury (not necessarily requiring neurological intervention). According to CCHR, head CT scanning is only required for patients with minor head injuries (defined as witnessed loss of consciousness, definite amnesia, or witnessed disorientation in a patient with a GCS score of 13-15) with any one of the following criterion: 
High risk (for neurological intervention)

1. GCS score $<15$ at 2 hours after injury

2. Suspected open or depressed skull fracture

3. Any sign of basal skull fracture (hemotympanum, 'raccoon' eyes, cerebrospinal fluid otorrhoea/rhinorrhoea, Battle's sign)

4. Vomiting (at least, two episodes)

5. Age $>65$ years

Medium risk (for brain injury on $\mathrm{CT}$ )

1. Amnesia before impact $>30 \mathrm{~min}$

2. Dangerous mechanism of head trauma (motor vehicle crash to pedestrian, occupant ejected from motor vehicle, fall from height $>3$ feet or five stairs).

The sensitivity and specificity of CCHR was reported as $100 \%$ and $50.6 \%-65.6 \%$, respectively.

\subsection{Abdul Latip et al. , 2004}

Abdul Latip et.al. conducted a cross-sectional study on $94 \mathrm{MHI}$ patients who were 12 years old and above, with a history of a blow to the head, and a GCS score of 13-15. They excluded the cases of known medical illnesses, suffered from cerebrovascular diseases or intracranial pathology, those who were on anti-coagulant medication, had a previous history of brain surgery, or facial bone fracture.

The positive outcome findings in this study were as follows: extradural, subdural, subarachnoid, intraparenchymal or intraventricular hemorrhage, pneumocephalus, cerebral contusion, midline shift or depressed skull fracture and linear vault fracture.

According to this decision rule, patients with a GCS of 13 or 14 and any of the following signs or symptoms will benefit from CT scanning: vomiting, craniofacial injuries, abnormal CNS findings, involvement in non-motor vehicle accidents, having abnormal CNS examination, craniofacial injury or skull fracture. Patients with GCS of 15 who have a skull fracture are also eligible for CT scanning.

\subsection{NEXUS II, 2005}

National Emergency X-Radiography Utilization Study II (NEXUS II) was a prospective study performed as a multi center cohort on 13728 patients of blunt head trauma to design and validate a decision rule to detect those cases of head trauma which are at very low risk of developing intracranial injury in order to reduce the number of ordered CT scanning (Mower et al., 2002, 2005). Patients with a history of blunt head trauma referred to 21 participating center were included. They underwent head CT scanning based on the decision of the treating physician, not based on the study protocol.

The positive outcome findings were as follows: mass effect, signs of herniation, basal cistern compression or midline shift, substantial epidural or subdural hematomas (greater than 1.0 $\mathrm{cm}$ in width, or causing mass effect), substantial cerebral contusion, extensive subarachnoid hemorrhage, hemorrhage in the posterior fossa, intraventricular hemorrhage, bilateral hemorrhage of any type, depressed or diastatic skull fracture, pneumocephalus, diffuse cerebral edema, diffuse axonal injury.

The study sample size was 13728 but MHI patients were about 2100 cases.

The sensitivity of this decision rule to identify the intra cranial injury in MHI patients was $95.2 \%(92.2 \%-97.2 \%)$ and its specificity was $17.3 \%(16.5 \%-18.0 \%)$. 
According to this guideline, the head CT scanning is needed for the patients presenting any of the following signs and symptoms:

1. Evidence of significant skull fracture,

2. Scalp hematoma,

3. Neurologic deficit,

4. Altered level of alertness,

5. Abnormal behavior,

6. Coagulopathy,

7. Persistent vomiting,

8. Age of 65 years or more

\subsection{CT in head injury patients (CHIP), 2007}

Smiths et al., compared and validated NOC and CCHR in 2005 by a prospective cohort study on 3181 patients. They showed that NOC has a higher sensitivity for cranial neural traumatic lesions or clinically significant finding, when used in patients with a GCS score of 13-15; while CCHR had more power to detect patients requiring neurosurgical intervention and hence would reduce the number of CT scan performed (Smith et al., 2005).

Two years later, Smith et al., developed their decision rule named "CT in head injury patients (CHIP)"

They included patients aged 16 years old and more who presented within 24 hours of blunt injury to the head, with a GCS score of 13 to 14 or a GCS score of 15 , with at least 1 of the following risk factors: history of loss of consciousness, short-term memory deficit, amnesia for the traumatic event, posttraumatic seizure, vomiting, severe headache, clinical evidence of intoxication with alcohol or drugs, use of anticoagulants or history of coagulopathy, external evidence of injury above the clavicles, and neurologic deficit.

The outcomes of interest were the intra cranial traumatic finding in CT scan (except for isolated linear skull fracture). The secondary outcome was need to neurosurgical intervention contingent to initial CT.

The study sample size was 3181 .

According to CHIP criteria, a CT scanning is indicated if one major criterion or 2 minor criteria exist.

Major criteria were as follow:

1. Pedestrian or cyclist versus vehicle

2. Ejected from vehicle

3. Vomiting

4. Post- traumatic amnesia $\geq 4 \mathrm{~h}$

5. Clinical signs of skull fracture (Any injury that suggests a skull fracture, such as palpable discontinuity of the skull, leakage of cerebrospinal fluid, raccoon eye bruising, and bleeding from the ear)

6. GCS score $<15$

7. GCS deterioration $\geq 2$ points (1 hour after presentation)

8. Use of anticoagulant therapy

9. Posttraumatic seizure

10. Age $\geq 60 \mathrm{y}$

Minor criteria were as follows:

1. Fall from any elevation 
2. Persistent antegrade amnesia (any deficit of short-term memory)

3. Posttraumatic amnesia of 2 to $4 \mathrm{~h}$

4. Contusion of the skull

5. Neurologic deficit

6. Loss of consciousness

7. GCS deterioration of 1 point (1 hour after presentation)

8. Age of $40-60$ years

CHIP prediction rule is of high sensitivity for selective use of CT in patients with minor head injury; it strongly identifies cases probable to have positive finding on CT and determines whether patients will need neurosurgery or not.

\subsection{Lee et al. criteria, 2009}

These criteria were developed based on a retrospective study on $898 \mathrm{MHI}$ patients.

Patients aged 16 years and more, presented with a history of blunt head trauma, who had a GCS score of 13-15 (with or without LOC) and admitted to the hospital for more than 12 hours were included.

The positive outcome was defined as all intracranial post-traumatic hematoma or contusion, depressed fractures, traumatic subarachnoid hemorrhage, and pneumocephalus. Isolated linear skull fracture and initial diagnosis of chronic subdural hematoma were not considered as abnormal CT findings.

They identified four sub-groups of patients subject to minor head injury according to the possibility of developing post- traumatic complications:

Very low risk: GCS score of 15 having no history of LOC or headache;

Low risk: GCS score of 15 with LOC and/or headache;

Medium risk: GCS score of 15 with a skull fracture, neurological deficits or with one or more of the mentioned risk factors;

High risk: GCS score of 15 with abnormal CT findings and GCS score of 14 and 13.

Of these four divided sub- groups, CT scan was advised for all the head injured cases; very low and low risk factors may be discharged; while medium and high risk patients of minor head injury should be admitted for close observation due to the risk of deterioration; in addition, high risk cases should be treated as moderate head injured. Patients younger than 16 years old or those with penetrating head trauma and hospital admission in less than 12 hours had been excluded from this retrospective study.

\subsection{Saadat et al., 2009}

In a cohort study held in Tehran University of Medical Sciences in Iran in 2004, a statistical model was developed for predicting the occurrence of intracranial lesions in patients with MHI (Saadat et al., 2006). This study was ordered by Iranian ministry of health and medical education. It was intended to be considered by rural physicians as a guideline, to select the MHI patients that need to be referred for head CT scanning. The decision rule was more developed later, using larger sample size, and the final model was published in 2009 (Saadat et al, 2009). This decision rule was based on 318 patients with a history of blunt head trauma and a Glasgow Coma Scale (GCS) score $\geq 13$ who had presented within 12 hours of trauma. Computed tomography findings that necessitated neurosurgical care (either observation or intervention) were considered as positive findings. This study considered broader intracranial findings on CT as an outcome measure including linear skull fracture as well as 
depressed, mastoid, comminuted, basilar, and sphenoid fracture; epidural, subdural, subarachnoid, intraparenchymal (including petechial), and intraventricular hemorrhage; brain contusion; pneumocephalus; and midline shift.

All MHI patients whose GCS score was 13-15, regardless of any other concomitant risk factors and LOC or amnesia, were included. Therefore, the results of this study could be applied to a broader spectrum of patients with minor head injury. The following patients were excluded from analysis: opium-addicted patients, those with concurrent major wounds or fractures that necessitated specialized care in a hospital, patients whose condition was unstable and who could not be safely transferred to the radiology department, patients suspected of malingering, and those patients who refused to participate in the study.

A logistic regression model was used to differentiate the subset of patients with minor head injury who may demonstrate intracranial lesions if they underwent brain CT scanning. According to this decision rule, MHI patients with 1 major criterion or 2 minor criteria need CT scanning and $13 \%$ of the cases represent positive intracranial lesion in head CT scan. A normal GCS score after a minor head injury did not guarantee the absence of significant neurological injury in this study, as $0.6 \%$ of such patients required neurosurgical intervention. The sensitivity and specify of this decision rule was reported as $100 \%$ and $46 \%$, respectively. The criteria were as follows:

Major criteria:

1. GCS score $<14$

2. Presence of the raccoon sign

3. Failure to remember the impact

4. Age $>65$ years

5. Vomiting after impact

Minor criteria:

1. Scalp wound

2. GCS score $<15$.

\section{CT scanning in childhood}

The indications of performing a CT in children are different from those applied in adults. This is partly due to the structure of skull and the neurological development of children. The concern with radiation to children is another reason for the difference of head CT scanning in childhood. It was estimated that the lifetime risk of cancer mortality, attributable to the ionizing radiation due to head CT scanning during the emergency admission, for a one-year-old child would be about 1 in 1500; while it is about 1 in 5000 for 10 years olds (Brenner et al., 2001). The national institute for Health and Clinical Excellence (NICE) has recommended immediate request for CT scan of head in children if there are any of the followings: Loss of consciousness lasting more than 5 minutes (witnessed), amnesia (antegrade or retrograde) lasting more than 5 minutes, abnormal drowsiness, three or more discrete episodes of vomiting, clinical suspicion of non-accidental injury, post-traumatic seizure but no history of epilepsy, GCS less than 14, or for a baby under 1 year GCS (pediatric) less than 15, on assessment in the emergency department, suspicion of open or depressed skull injury or tense fontanel, any sign of basal skull fracture (haemotympanum, 'panda' eyes, cerebrospinal fluid leakage from the ear or nose, Battle's sign), focal 
neurological deficit, if under 1 year, presence of bruise, swelling or laceration of more than 5 $\mathrm{cm}$ on the head, dangerous mechanism of injury (high-speed road traffic accident either as pedestrian, cyclist or vehicle occupant, fall from a height of greater than $3 \mathrm{~m}$, high-speed injury from a projectile or an object). This guideline is not limited to MHI patients however. There are decision rules which recommend a set of findings in performing CT for children of traumatic cases when they are subjected to head trauma.

\subsection{Children's head injury algorithm for the prediction of important clinical events (CHALICE); Dunning et al., 2006}

This decision rule suggests a set of criteria based on history, physical examination findings and the mechanism of trauma to order a head CT scanning for MHI children. The criteria are as follows:

\section{History}

1. Witnessed LOC of $>5$ min duration

2. History of amnesia (either antegrade or retrograde) of $>5$ min duration

3. Abnormal drowsiness (defined as drowsiness in excess of that expected by the examining doctor)

4. $\geq 3$ vomits after head injury (a vomit is defined as a single discrete episode of vomiting)

5. Suspicion of non accidental injury (defined as any suspicion of non accidental injury by the examining doctor)

6. Seizure after head injury in a patient who has no history of epilepsy

\section{Examination}

7. GCS less than 14, or GCS less than 15 if the patient is younger than one year old

8. Suspicion of penetrating or depressed skull injury or tense fontanel

9. Sign of basal skull fracture (defined as evidence of blood or cerebrospinal fluid leakage from the ear or nose and panda eyes)

10. Battle's sign, haemotympanum, facial crepitus or serious facial injury)

11. Positive focal neurological sign (defined as any focal neurological sign, including motor, sensory, coordination or reflex abnormality)

12. Presence of bruise, swelling or laceration $>5 \mathrm{~cm}$ if the patient is younger than one year old

Mechanism

13. High-speed road traffic accident either as a pedestrian, cyclist or occupant (defined as accident with speed $>40 \mathrm{~m} / \mathrm{h}$ or $64 \mathrm{~km} / \mathrm{h}$ )

14. Fall of more than 3 meters in height

15. High-speed injury from a projectile or an object

A CT scan is required if any of the above criteria are present

\subsection{Canadian Assessment of Tomography for Childhood Head (CATCH), 2010}

It was the name that Osmond et al. gave to their clinical decision rule for the use of computed tomography in children with minor head injury.

They included children aging between 0-16 years old, subject to blunt head trauma within 24 hours, with a GCS score of 13 to 15, with loss of consciousness, disorientation, amnesia, persistent irritability or vomiting. Exclusion was done if the cases were of obvious penetrating injury or depressed skull fracture, acute focal neurologic deficits, chronic 
developmental delay generally, suspected child abuse leading to such head trauma, and if they were referred for reassessment of previously treated head injury.

They studied 3866 cases and examined 2043 cases by CT scanning.

According to this decision rule a head CT scan is necessary if the child has a minor head trauma (defined as head injury within the past 24 hours associated with loss of consciousness, amnesia, disorientation, persistent vomiting (more than one episode) or persistent irritability (in a child under two years of age) in a patient with GCS score of 13 15) and one of the following criteria:

1. GCS score lower than 15 two hours post trauma

2. Possible open or depressed skull fracture

3. Irritability

4. Worsening headache

The above signs and symptoms identify patients with High risk in whom neurosurgery intervention is unavoidable

The following criteria are indication for CT scanning as well and they identify patients with Medium risk in whom brain injury on CT scanning (not necessarily requiring neurological intervention) is probable:

5. "Raccoon" eyes, otorrhea or rhinorrhea of cerebrospinal fluid, hemotympanum, Battle's sign or other signs suggesting basal skull fracture

6. Large and boggy hematoma of the scalp

7. Dangerous mechanism of injury (e.g. fall from $>3 \mathrm{ft}[91 \mathrm{~cm}]$ or higher than 5 stairs, or motor cycle rider with no helmet)

The Sensitivity and specificity of this decision rule was reported $100 \%$ and $70.2 \%$, respectively. When all these seven risk factors were considered, $51.9 \%$ of patients would be eligible for CT scanning.

\section{Conclusion}

There are several decision rules published in the literature. Adherence to a decision rule will result in uniformity in ordering CT scan. However, there are considerations on selection of a decision rule:

- External validity: The study subjects enrolled in different studies have not been totally similar. The inclusion/exclusion criteria used in the specific study, determines its external validity, that is, what population of patients will benefit from that specific decision rule. For example, Miller's criteria and NOC are developed based on patients who had presented with a GCS score of 15 and a history of LOC, while patients studied for CCHR, CHIP, Lee et al and Saadat et al included patients with GCS 13 and more. Therefore, Miller's and NOC criteria may not be generalizable to patients who are presented with a GCS score of 13-14. Similarly, while Saadat et al criteria are applicable to patients with and without LOC, this may not be the case with the decision rules that excluded patients who do not represent with LOC.

On the other hand, a wide inclusion criteria may affect the internal validity of a study; therefore, a decision rule that is developed based on a heterogeneous group of patients may be less reliable than criteria that are developed by focusing on a homogenous, well defined group of patients. It is best applicable however, to the same defined group of patients. 
- Positive outcome: CCHR excluded patients with obvious depressed skull fracture while NEXUS II considered depressed skull fracture as a positive outcome. Isolated linear skull fracture was not considered a positive outcome in CHIP criteria and Lee et al study, but it was considered so in Abdul Latip et al. study.

While adopting a decision rule, one should note the capabilities of the health facility setting to manage the consequences of a missed lesion. If a neurosurgeon is available in the health facility, then missing a lesion that could be safely managed later, may not be that serious. On the other hand, if management of a missed lesion is not possible in the given health facility, it would be better to adhere to decision rules that are designed to detect a wide range of outcomes.

- Age group: NOC included patients 3 years and older while CCHR, CHIP, Lee et al and Saadat et al included patients 16 years and older and Abdul Latip et al. included patients aged 12 and more. Therefore, these latter criteria should not be applied to children.

Almost all published criteria are developed based on the assumption that the predictive power of the decision rules is the same across the age groups included in the study. While this remains to be studied specifically, equal distribution of cases in the study group may promise similar predictive value in all ages. This is of special importance in the case of elderly patients.

- Sample size: as a rule, the larger sample size is expected to give a more reliable prediction rule, provided that a reasonable proportion of cases represent positive outcome. Therefore, among the studies that have prepared CT scan from all the included patients, a greater sample size results in better prediction rule.

- Inter-rater agreement: the criteria will be used by different practicing physicians. They may have different perception from a single criterion. For example, the inter-observer agreement of physicians on the CCHR criteria is reported to be higher than NOC (stiell e. al. 2005).

The inter-observer reliability of physicians should be measured and considered before adopting a specific decision rule.

- Sensitivity and specificity: The sensitivity and specificity of decision rules are described by their authors; however, the external validation studies generally provide less promising estimates. The higher sensitivity will result in less risk of missing a positive case. Generally, the higher sensitivity means less specificity that in turn results in less reduction of unnecessary CT scans. One should take in mind that these indices should be interpreted in conjunction with the "Positive outcome definition" of a specific study. A decision rule may appear more sensitive simply because it did not mean to detect some intracranial outcomes that another decision rule intended to do so.

In external validation studies, CCHR and the NOC provided with similarly high sensitivity to detect serious neurological outcomes in MHI patients with GCS $=15$; however, the specificity of the CCHR was higher than the NOC. Therefore, application of the CCHR would result in lower use of CT imaging (stiell e. al. 2005). In patients aged 1 to 20 years, the sensitivity of NOC was reported higher than NEXUS II and CCHR. The specificity of CCHR was higher than NEXUS II and it was higher than NOC.

- To order or not to order? While decision rules are developed to reduce unnecessary CT scanning, they are more reliable when used to detect the patients who need a CT rather than exclude a patient from scanning. The reason is the limited number of positive cases 
that the models are based on them. Note that deriving a decision rule is different from fitting a model for a cause-effect relationship. Both the indications used in decision rules and the intracranial lesions are results of another cause, the trauma. The statistical models just try to find visible signs and symptoms that are indicative of intracranial injury and there is no guarantee that there is no lesion if none of the visible signs or symptoms is present.

In some of the above mentioned studies, all patients did not undergo CT scanning but based on the judgment of the treating physician. If all patients are not studied by CT scan, there is no guarantee that they did not benefit from CT scanning. In fact, this limits the advantage of big sample size of NEXUS II study as it included the large number of patients whom physicians decided to order CT. Validity of this criteria for MHI patients who did not undergo CT scan is to be studied.

\section{Future studies}

There is need for further studies to cover the following issues:

- The optimal time of the first CT scanning in MHI patients needs to be studied. The predictive value of a single CT may depend on the time elapsed since the trauma. This needs to be studied specifically.

- A single CT scan may not reveal any significant intracranial finding; however, clinicians may order another brain scan according to the clinical presentations of the patient. The optimum time interval that is required to pass before re-ordering a CT scan needs to be studied.

- There is variability in the positive outcome definition. Medical centers need to assure detection of some important intracranial lesions. The important lesion may not mean the same for different settings (e.g. rural clinics versus different levels of trauma centers).

\section{References}

NICE. Head injury: Triage, assessment, investigation and early management of head injury in infants, children and adults [online]. Available at: http:/ / www.nice.org.uk

Abdul Latip L.S., Ahmad Alias N.A., Ariff aR., et al. (2004). CT scan in minor head injury: a guide for rural doctors. J Clin Neurosci, Vol. 11, No. 8, (Nov), pp 835-839, ISSN 0967-5868 (Print)

Af Geijerstam J.L. \& Britton M. (2003). Mild head injury - mortality and complication rate: meta-analysis of findings in a systematic literature review. Acta Neurochir (Wien), Vol. 145, No. 10, (Oct), pp 843-850; discussion 850, ISSN 0001-6268 (Print)

Berrington De Gonzalez A. \& Darby S. (2004). Risk of cancer from diagnostic X-rays: estimates for the UK and 14 other countries. Lancet, Vol. 363, No. 9406, (Jan 31), pp 345-351, ISSN 1474-547X (Electronic)

Borczuk P. (1995). Predictors of intracranial injury in patients with mild head trauma. Ann Emerg Med, Vol. 25, No. 6, (Jun), pp 731-736, ISSN 0196-0644 (Print)

Bordignon K.C. \& Arruda W.O. (2002). CT scan findings in mild head trauma: a series of 2,000 patients. Arq Neuropsiquiatr, Vol. 60, No. 2-A, (Jun), pp 204-210, ISSN 0004282X (Print) 
Borg J., Holm L., Cassidy J.D., et al. (2004). Diagnostic procedures in mild traumatic brain injury: results of the WHO Collaborating Centre Task Force on Mild Traumatic Brain Injury. J Rehabil Med, Vol. No. 43 Suppl, (Feb), pp 61-75, ISSN 1650-1977 (Print)

Brenner D., Elliston C., Hall E., et al. (2001). Estimated risks of radiation-induced fatal cancer from pediatric CT. AJR Am J Roentgenol, Vol. 176, No. 2, (Feb), pp 289-296, ISSN 0361-803X (Print)

Brix G., Nissen-Meyer S., Lechel U., et al. (2009). Radiation exposures of cancer patients from medical X-rays: how relevant are they for individual patients and population exposure? Eur J Radiol, Vol. 72, No. 2, (Nov), pp 342-347, ISSN 1872-7727 (Electronic)

Cassidy J.D., Carroll L.J., Peloso P.M., et al. (2004). Incidence, risk factors and prevention of mild traumatic brain injury: results of the WHO Collaborating Centre Task Force on Mild Traumatic Brain Injury. J Rehabil Med, Vol. No. 43 Suppl, (Feb), pp 28-60, ISSN 1650-1977 (Print)

Dunning J., Batchelor J., Stratford-Smith P., et al. (2004). A meta-analysis of variables that predict significant intracranial injury in minor head trauma. Archives of Disease in Childhood, Vol. 89, No. 7, (July 1, 2004), pp 653-659, 1468-2044 (Electronic)

Dunning J., Daly J.P., Lomas J.P., et al. (2006). Derivation of the children's head injury algorithm for the prediction of important clinical events decision rule for head injury in children. Arch Dis Child, Vol. 91, No. 11, (Nov), pp 885-891, ISSN 14682044 (Electronic)

Einstein aJ., Henzlova M. J.\& Rajagopalan S. (2007). Estimating risk of cancer associated with radiation exposure from 64-slice computed tomography coronary angiography. JAMA, Vol. 298, No. 3, (Jul 18), pp 317-323, ISSN 1538-3598 (Electronic)

Fabbri A., Servadei F., Marchesini G., et al. (2004). Prospective validation of a proposal for diagnosis and management of patients attending the emergency department for mild head injury. J Neurol Neurosurg Psychiatry, Vol. 75, No. 3, (Mar), pp 410-416, ISSN 0022-3050 (Print)

Graham I.D., Stiell I.G., Laupacis A., et al. (1998). Emergency Physicians' Attitudes toward and Use of Clinical Decision Rules for Radiography. Academic Emergency Medicine, Vol. 5, No. 2, pp 134-140, ISSN 1553-2712

Hall E.J. \& Brenner D.J. (2008). Cancer risks from diagnostic radiology. Br J Radiol, Vol. 81, No. 965, (May), pp 362-378, ISSN 1748-880X (Electronic)

Harad F.T. \& Kerstein M.D. (1992). Inadequacy of bedside clinical indicators in identifying significant intracranial injury in trauma patients. J Trauma, Vol. 32, No. 3, (Mar), pp 359-361; discussion 361-353, ISSN 0022-5282 (Print)

Haydel M.J. (2005). Clinical decision instruments for CT scanning in minor head injury. JAMA, Vol. 294, No. 12, (Sep 28), pp 1551-1553, ISSN 1538-3598 (Electronic)

Haydel M.J., Preston C.A., Mills T.J., et al. (2000). Indications for computed tomography in patients with minor head injury. N Engl J Med, Vol. 343, No. 2, (Jul 13), pp 100-105, ISSN 0028-4793 (Print)

Holmes J.F., Hendey G.W., Oman J.A., et al. (2006). Epidemiology of blunt head injury victims undergoing ED cranial computed tomographic scanning. Am J Emerg Med, Vol. 24, No. 2, (Mar), pp 167-173, ISSN 0735-6757 (Print) 
Jager T.E., Weiss H.B., Coben J.H., et al. (2000). Traumatic Brain Injuries Evaluated in U.S. Emergency Departments, 1992-1994. Academic Emergency Medicine, Vol. 7, No. 2, pp 134-140, ISSN 1553-2712

Jeret J.S, Mandell M., Anziska B., et al. (1993). Clinical predictors of abnormality disclosed by computed tomography after mild head trauma. Neurosurgery, Vol. 32, No. 1, (Jan), pp 9-15; discussion 15-16, ISSN 0148-396X (Print)

Katada K. (2006). [CT radiation exposure in Japan: analysis of social factors]. Nippon Hoshasen Gijutsu Gakkai Zasshi, Vol. 62, No. 5, (May 20), pp 649-656, ISSN 03694305 (Print)

Klauber M.R., Marshall L.F., Luerssen T.G., et al. (1989). Determinants of head injury mortality: importance of the low risk patient. Neurosurgery, Vol. 24, No. 1, (Jan), pp 31-36, ISSN 0148-396X (Print)

Kraus J.F. \& Nourjah P. (1988). The epidemiology of mild, uncomplicated brain injury. J Trauma, Vol. 28, No. 12, (Dec), pp 1637-1643, ISSN 0022-5282 (Print)

Langlois Ja., Rutland-Brown W. \& Ke. T. (2006). Traumatic Brain Injury in the United States: Emergency Department Visits, Hospitalizations, and Deaths. Centers for Disease Control and Prevention, National Center for Injury Prevention and Control

Langlois Ja., Rutland-Brown W. \& Ke. T. (2004). Traumatic Brain Injury in the United States: Emergency Department Visits, Hospitalizations, and Deaths. Centers for Disease Control and Prevention, National Center for Injury Prevention and Control

Lee Y.B. \& Kwon S.J. (2009). A more detailed classification of mild head injury in adults and treatment guidelines. J Korean Neurosurg Soc, Vol. 46, No. 5, (Nov), pp 451-458, ISSN 1598-7876 (Electronic)

Mccaig L.F. \& Burt C.W. (2001). National Hospital Ambulatory Medical Care Survey: 1999 emergency department summary. Adv Data, Vol. No. 320, (Jun 25), pp 1-34, ISSN 0147-3956 (Print)

Menegazzi J.J., Davis E.A., Sucov aN., et al. (1993). Reliability of the Glasgow Coma Scale when used by emergency physicians and paramedics. J Trauma, Vol. 34, No. 1, (Jan), pp 46-48, ISSN 0022-5282 (Print)

Mettler Fa, Jr.Wiest, P.W., Locken J.A., et al. (2000). CT scanning: patterns of use and dose. J Radiol Prot, Vol. 20, No. 4, (Dec), pp 353-359, ISSN 0952-4746 (Print)

Miller E.C., Holmes J.F. \& Derlet R.W. (1997). Utilizing clinical factors to reduce head CT scan ordering for minor head trauma patients. J Emerg Med, Vol. 15, No. 4, (JulAug), pp 453-457, ISSN 0736-4679 (Print)

Mower W.R., Hoffman J.R., Herbert M., et al. (2002). Developing a clinical decision instrument to rule out intracranial injuries in patients with minor head trauma: methodology of the NEXUS II investigation. Ann Emerg Med, Vol. 40, No. 5, (Nov), pp 505-514, ISSN 0196-0644 (Print)

Mower W.R., Hoffman J.R., Herbert M., et al. (2005). Developing a decision instrument to guide computed tomographic imaging of blunt head injury patients. J Trauma, Vol. 59, No. 4, (Oct), pp 954-959, ISSN 0022-5282 (Print)

Norwood S.H., Mcauley C.E., Berne J.D., et al. (2002). A prehospital glasgow coma scale score $<$ or $=14$ accurately predicts the need for full trauma team activation and patient hospitalization after motor vehicle collisions. J Trauma, Vol. 53, No. 3, (Sep), pp 503-507, ISSN 0022-5282 (Print) 
Osmond M.H., Klassen T.P., Wells G.A., et al. (2010). CATCH: a clinical decision rule for the use of computed tomography in children with minor head injury. CMAJ, Vol. 182, No. 4, (Mar 9), pp 341-348, ISSN 1488-2329 (Electronic)

Rimel R.W., Giordani B., Barth J.T., et al. (1981). Disability caused by minor head injury. Neurosurgery, Vol. 9, No. 3, (Sep), pp 221-228, ISSN 0148-396X (Print)

Saadat S., Ghodsi S.M., Holakouei Naeini K., et al. (2006). A statistical model for predicting the occurrence of intracranial lesions in patients with mild head trauma. PAYESH JULY, Vol. 5, No. 3, pp 193-200

Saadat S., Ghodsi S.M., Naieni K.H., et al. (2009). Prediction of intracranial computed tomography findings in patients with minor head injury by using logistic regression. J Neurosurg, Vol. 111, No. 4, (Oct), pp 688-694, ISSN 1933-0693 (Electronic)

Schynoll W., Overton D., Krome R., et al. (1993). A prospective study to identify high-yield criteria associated with acute intracranial computed tomography findings in headinjured patients. Am J Emerg Med, Vol. 11, No. 4, (Jul), pp 321-326, ISSN 0735-6757 (Print)

Shackford S.R., Wald S.L., Ross S.E., et al. (1992). The clinical utility of computed tomographic scanning and neurologic examination in the management of patients with minor head injuries. J Trauma, Vol. 33, No. 3, (Sep), pp 385-394, ISSN 00225282 (Print)

Smits M., Dippel D.W., De Haan G.G., et al. (2005). External validation of the Canadian CT Head Rule and the New Orleans Criteria for CT scanning in patients with minor head injury.JAMA, Vol. 294, No. 12, (Sep 28), pp 1519-1525, ISSN 1538-3598 (Electronic)

Smits M., Dippel D.W., Steyerberg E.W., et al. (2007). Predicting intracranial traumatic findings on computed tomography in patients with minor head injury: the CHIP prediction rule. Ann Intern Med, Vol. 146, No. 6, (Mar 20), pp 397-405, ISSN 15393704 (Electronic)

Stein S.C. \& Ross S.E. (1990). The value of computed tomographic scans in patients with low-risk head injuries. Neurosurgery, Vol. 26, No. 4, (Apr), pp 638-640, ISSN 0148396X (Print)

Stein S.C. \& Ross S.E. (1992). Minor head injury: a proposed strategy for emergency management. Ann Emerg Med, Vol. 22, No. 7, (Jul), pp 1193-1196, ISSN 0196-0644 (Print)

Stiell I.G., Clement C.M., Rowe B.H., et al. (2005). Comparison of the Canadian CT Head Rule and the New Orleans Criteria in patients with minor head injury. JAMA, Vol. 294, No. 12, (Sep 28), pp 1511-1518, ISSN 1538-3598 (Electronic)

Stiell I.G., Lesiuk H., Wells G.A, et al. (2001). Canadian CT head rule study for patients with minor head injury: methodology for phase II (validation and economic analysis). Ann Emerg Med, Vol. 38, No. 3, (Sep), pp 317-322, ISSN 0196-0644 (Print)

Stiell I.G., Lesiuk H., Wells G.A., et al. (2001). The Canadian CT Head Rule Study for patients with minor head injury: rationale, objectives, and methodology for phase I (derivation). Ann Emerg Med, Vol. 38, No. 2, (Aug), pp 160-169, ISSN 0196-0644 (Print)

Stiell I.G., Wells G.A.,Vandemheen K., et al. (2001). The Canadian CT Head Rule for patients with minor head injury. Lancet, Vol. 357, No. 9266, (May 5), pp 1391-1396, ISSN 0140-6736 (Print) 


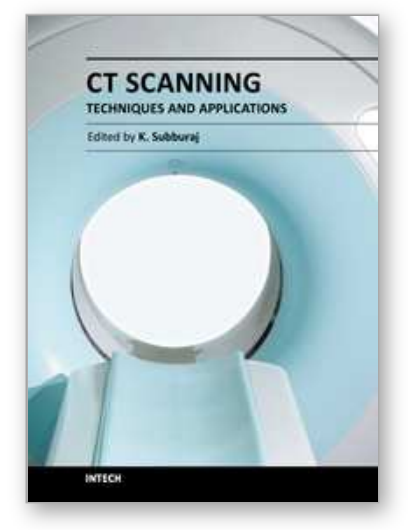

\author{
CT Scanning - Techniques and Applications \\ Edited by Dr. Karupppasamy Subburaj
}

ISBN 978-953-307-943-1

Hard cover, 348 pages

Publisher InTech

Published online 30, September, 2011

Published in print edition September, 2011

Since its introduction in 1972, X-ray computed tomography (CT) has evolved into an essential diagnostic imaging tool for a continually increasing variety of clinical applications. The goal of this book was not simply to summarize currently available CT imaging techniques but also to provide clinical perspectives, advances in hybrid technologies, new applications other than medicine and an outlook on future developments. Major experts in this growing field contributed to this book, which is geared to radiologists, orthopedic surgeons, engineers, and clinical and basic researchers. We believe that CT scanning is an effective and essential tools in treatment planning, basic understanding of physiology, and and tackling the ever-increasing challenge of diagnosis in our society.

\title{
How to reference
}

In order to correctly reference this scholarly work, feel free to copy and paste the following:

Saeed Shoar and Soheil Saadat (2011). CT Scanning in Minor Head Injury, CT Scanning - Techniques and Applications, Dr. Karupppasamy Subburaj (Ed.), ISBN: 978-953-307-943-1, InTech, Available from: http://www.intechopen.com/books/ct-scanning-techniques-and-applications/ct-scanning-in-minor-head-injury

\section{INTECH}

open science | open minds

\section{InTech Europe}

University Campus STeP Ri

Slavka Krautzeka 83/A

51000 Rijeka, Croatia

Phone: +385 (51) 770447

Fax: +385 (51) 686166

www.intechopen.com

\section{InTech China}

Unit 405, Office Block, Hotel Equatorial Shanghai

No.65, Yan An Road (West), Shanghai, 200040, China

中国上海市延安西路65号上海国际贵都大饭店办公楼 405 单元

Phone: +86-21-62489820

Fax: $+86-21-62489821$ 
(C) 2011 The Author(s). Licensee IntechOpen. This chapter is distributed under the terms of the Creative Commons Attribution-NonCommercialShareAlike-3.0 License, which permits use, distribution and reproduction for non-commercial purposes, provided the original is properly cited and derivative works building on this content are distributed under the same license. 